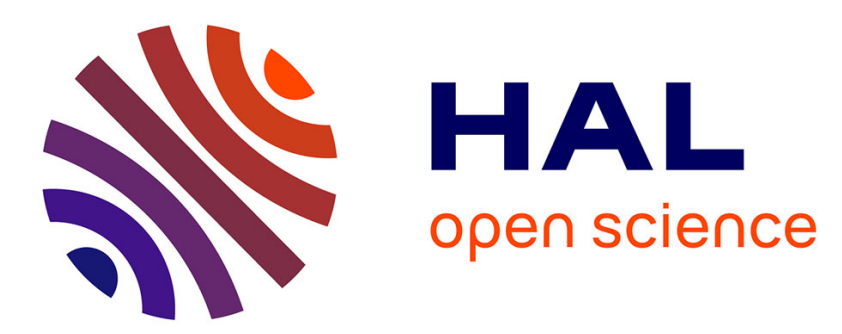

\title{
Amélioration des performances du récepteur infrarouge pneumatique du type de Golay
}

\author{
J.-M. Munier, A. Hadni
}

\section{To cite this version:}

J.-M. Munier, A. Hadni. Amélioration des performances du récepteur infrarouge pneumatique du type de Golay. Revue de Physique Appliquée, 1970, 5 (6), pp.845-852. 10.1051/rphysap:0197000506084500 . jpa-00243464

\section{HAL Id: jpa-00243464 https://hal.science/jpa-00243464}

Submitted on 1 Jan 1970

HAL is a multi-disciplinary open access archive for the deposit and dissemination of scientific research documents, whether they are published or not. The documents may come from teaching and research institutions in France or abroad, or from public or private research centers.
L'archive ouverte pluridisciplinaire HAL, est destinée au dépôt et à la diffusion de documents scientifiques de niveau recherche, publiés ou non, émanant des établissements d'enseignement et de recherche français ou étrangers, des laboratoires publics ou privés. 


\title{
AMÉLIORATION DES PERFORMANCES DU RÉCEPTEUR INFRAROUGE PNEUMATIQUE DU TYPE DE GOLAY
}

\author{
par J.-M. MUNIER et A. HADNI \\ Institut de Physique, Université de Nancy, Nancy, France
}

(Reçu le 5 août 1970)

\begin{abstract}
Résumé. - Description d'une méthode de préparation des membranes absorbante et réfléchissante d'un récepteur pneumatique du type de Golay, du remplissage en xénon de la cellule de mesure et des résultats obtenus : fiabilité accrue et NEP $\simeq 2 \times 10^{-11} \mathrm{~W} . \mathrm{cps}^{-1 / 2}$.

Abstract. - Description of a method for preparing absorbing and reflecting membranes in a Golay type pneumatic detector, and for filling the cell with xenon. Discussion of the results : higher fiability, and NEP $\simeq 2 \times 10^{-11} \mathrm{~W} \cdot \mathrm{cps}^{-1 / 2}$.
\end{abstract}

I. Introduction. - I. 1 RAPPEL DESCRIPTIF (Fig. 1). - Le récepteur est constitué par une cavité contenant du xénon sous une pression déterminée. Cette cavité comporte à l'avant une membrane absorbante. Elle est fermée à l'arrière par une autre membrane dont les déformations servent à mesurer la quantité de chaleur absorbée par la cavité (1). L'amélioration $\mathrm{du}$ récepteur est conditionnée par la qualité de ces deux membranes. La description des procédés utilisés constitue l'essentiel de ce travail.

I. 2 PRINCIPE DE FONCTIONNEMENT DU RÉCEPTEUR DU TYPE DE GolAY. - La figure $2 a$ donne le schéma de la mesure optique de la déformation de la membrane. Supposons que l'axe optique du ménisque passe par le bord inférieur d'un trait noir de la grille $G$, son image se formera sur le trait blanc qui lui est symétrique. Si la membrane n'est plus plane, elle se comportera comme un miroir sphérique et l'image $\mathrm{A}^{\prime \prime} \mathbf{B}^{\prime \prime}$ n'est plus exactement superposée à l'objet $\mathrm{AB}$ (Fig. 2c). L'expression du grossissement s'écrit: $G=\alpha^{\prime} / \alpha$, avec $\alpha=\mathrm{AB} / \mathrm{F}$. On a $G=-1$ avec un miroir plan où $\alpha^{\prime}=-\alpha$ (méthode d'opposition).

Dans le cas où le miroir n'est plus plan, l'image $A^{\prime} B^{\prime}$ de $A B$ se forme au foyer de $M$ et joue le rôle d'objet virtuel pour $L$ qui en donne l'image $A^{\prime \prime} B^{\prime \prime}$ :
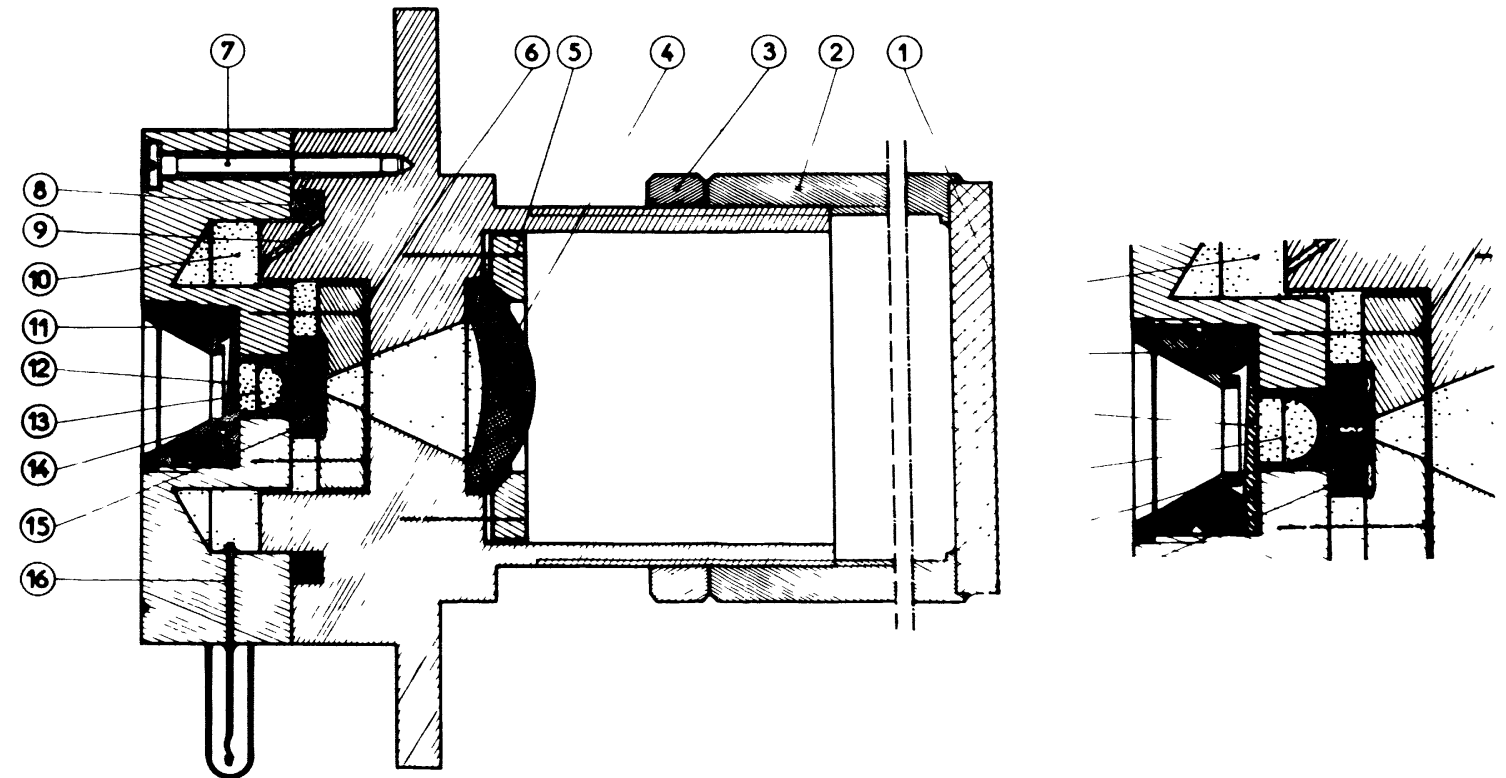

FIG. 1. - Plan du récepteur pneumatique : (1) Grille; (2) Support de la grille ; (3) Contre-écrou ; (4) Ménisque convergent ; (5) (6) (7) Vis d'assemblage ; (8) Joint torique d'étanchéité ; (9) Canal d'évacuation du gaz résiduel autour du joint ; (10) Ballast annulaire ; (11) Joint torique; (12) Fenêtre taillée en coin ; (13) Membrane absorbante; (14) membrane réfléchissante; (15) Fuite : intérieur-ballast; (16) Capillaire de remplissage. 

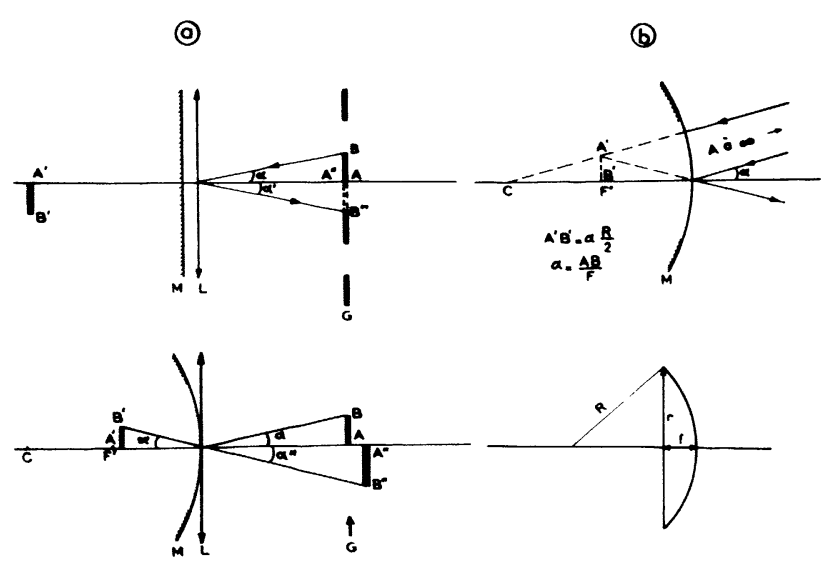

(c)

(d)

Fig. 2. - Schémas optiques : (a) Membrane non déformée (plane); (b) image d'un objet à l'infini donné par un miroir convexe; $(c)$ membrane déformée (ici convexe); $(d)$ flèche correspondant à la déformation.

$p, p^{\prime}$ et $F$ sont les distances de $\mathrm{AB}, \mathrm{A}^{\prime} \mathrm{B}^{\prime}$ à $\mathrm{L}$ et sa distance focale :

$$
p^{\prime}=\frac{p F}{p-F}
$$

d'où

$$
p^{\prime}=-\frac{R}{2} \frac{F}{-\frac{R}{2}-F}
$$

et

$$
\frac{\mathrm{A}^{\prime \prime} \mathrm{B}^{\prime \prime}}{\mathrm{A}^{\prime} \mathrm{B}^{\prime}}=\frac{F}{-\frac{R}{2}-F}=G,
$$

comme $\alpha=\mathrm{AB} / F$ et $\mathrm{A}^{\prime} \mathrm{B}^{\prime}=\alpha \cdot R / 2$, on obtient

$$
\mathrm{A}^{\prime} \mathrm{B}^{\prime}=\frac{\mathrm{AB}}{F} \frac{R}{2},
$$

d'où

$$
\mathrm{A}^{\prime \prime} \mathrm{B}^{\prime \prime}=\frac{F}{-\frac{R}{2}-F} \cdot \frac{\mathrm{AB}}{F} \cdot \frac{R}{2},
$$

donc

$$
\frac{\mathrm{A}^{\prime \prime} \mathrm{B}^{\prime \prime}}{\mathrm{AB}} \quad \frac{\frac{R}{2}}{-\frac{R}{2}-F}=\frac{1}{-1-\frac{2 F}{R}}=\frac{-1}{1+\frac{2 F}{R}}
$$

or $F$ est très petit devant $R, F=100 / 33,3=3 \mathrm{~cm}$.

On peut écrire

$$
G=\frac{\mathrm{A}^{\prime \prime} \mathrm{B}^{\prime \prime}}{\mathrm{AB}} \simeq-1+\frac{2 F}{R}=-1+\Delta G
$$

L'image $A^{\prime \prime} B^{\prime \prime}$ de $A B$ est de sens contraire et son grandissement varie de $\Delta G=2 F / R$. La variation de flux lumineux correspondante est proportionnelle à $[G]^{2}$, soit

$$
\Delta \varnothing \simeq 2 \Delta G
$$

$\Delta G$ étant très petit.

La flèche de la membrane est extrêmement faible, de l'ordre de $1 \AA$, le rayon de courbure du miroir correspondant est très grand (Fig. $2 d), R^{2}=r^{2}(R-f)^{2}$, mais $r^{2} \# r^{2} / 2 f$, avec $f=1 \AA, r=1 \mathrm{~mm}$. Nous avons donc $R=10^{14} / 2 \AA$, soit $5 \mathrm{~km}$.

On entrevoit la grande précision avec laquelle doit être faite cette membrane.

Calculons la relation liant la variation de pression interne $\mathrm{du}$ récepteur à celle du flux lumineux à travers la grille. La membrane peut être considérée comme une partie de sphère, telle une bulle de savon, nous avons : $\Delta p=4 \mathfrak{A} / R, \mathcal{A}$ est la tension superficielle du film ; $R=4 \mathfrak{t} / \Delta p$, or $R=r^{2} / 2 f$ et $f=r^{2} / 2 R$, donc $f=r^{2} / 8$. $\Delta p$. Par conséquent

$$
f=\frac{r^{2}}{8 \mathcal{A}} \frac{P}{T} \Delta T
$$

Nous avons montré plus haut que $\Delta \varnothing=4 F / R$ ce qui peut alors s'écrire

$$
\Delta \varnothing=\frac{4 F}{4 \mathfrak{A}} \Delta P
$$

ou encore

$$
\Delta \varnothing=\frac{F}{\mathcal{A}} \Delta P
$$

or $\Delta P / P=\Delta T / T$, soit

$$
\Delta \varnothing_{\text {lumineux }}=\frac{F}{\mathfrak{t}} P \frac{\Delta T}{T} .
$$

Cette expression représente la variation de flux lumineux relative à l'image d'un seul trait de la grille par rapport à son objet.

Quand on s'éloigne du centre de la membrane (supposons ce centre non éclairé, c'est-à-dire qu'il est vu sombre depuis la cellule photo-électrique), l'éclairement de la membrane va varier progressivement dans une direction perpendiculaire aux traits de la grille. En effet le diamètre de la membrane qui est parallèle aux traits de la grille est axe de symétrie pour les variations d'éclairement de la membrane. Plaçons-nous dans le cas où l'axe optique du ménisque passe par la ligne de séparation entre un trait opaque et un trait transparent. La membrane paraît uniformément sombre au repos, aucune lumière ne parvient à la cellule. Une faible déformation de la membrane fait varier les dimensions de l'image $A^{\prime \prime} B^{\prime \prime}$ de $A B$ et elle n'est plus superposable à l'élément de grille correspondant, la différence sera donnée par le rapport $\delta b / b=2 F / R$, ceci pour le trait objet d'ordre 1 . Pour le trait d'ordre $n$, l'écart en position sera $n . \delta b=2(F / R) . n . b$. Un trait sombre d'ordre $q$ se formera entièrement sur un trait sombre (de même pour un trait transparent). En cet endroit toute la lumière 
provenant d"un trait objet transparent repassera par un trait transparent :

$$
q(2 F R) \dot{b}=b-\delta b . \text { d’où } q=(R / 2 F)-1 .
$$

mais $R \gg 2 F$. Ceci se passera donc pour le trait dordre $q \# R / 2 F$.

La figure $3 a$ représente la série (numéros impairs) des rectangles élémentaires dont la somme est proportionnelle à léclairement apparent de la grille. Ainsi du Centre $\mathrm{O}$ (qui paraît noir) jusquà la distance $(q+1) b$, la quantité de lumière traversant la grille croît linéairement d'une valeur $E_{\min }=\varnothing L \delta b$ à une valeur $E_{\max }=\varnothing L(b-\delta b)$, où $L$ est l'unité de longueur d'un trait de grille.

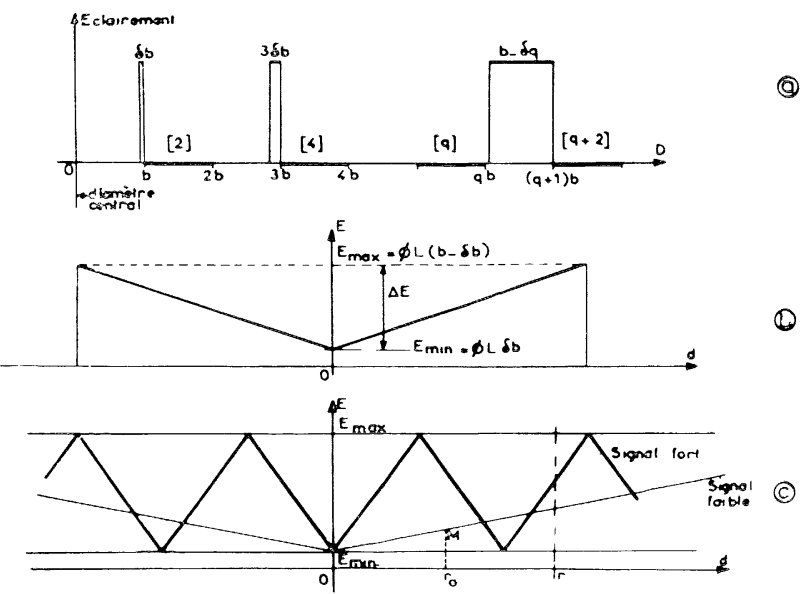

FIG. 3a. - Eclairement de la grille à partir du diamètre central.

Fig. 3b. - Eclairement apparent de la membrane à partir de son diamètre, parallèle aux traits de la grille.

FIG. 3c. Eclairement apparent de la membrane pour des déformations variables.

La figure $3 b$ représente graphiquement l'éclairement apparent de la membrane. Ceci suppose que la diffraction due à la grille est négligeable (le phénomène est bien visible sur les photographies de la figure $4 a, b)$.

La figure $3 c$ montre l'éclairement apparent de la membrane pour des déformations croissantes de celle-ci. Ces courbes sont théoriques et valables pour une membrane carrée et très grande. Le point $M$ est le point d’abscisse $r_{0}$ qui est focalisé sur la cellule photo-électrique. Nous pouvons aussi remarquer sur les figures $4 a$ et $4 b$, l'analogie frappante entre la courbe théorique (Fig. 3c) d'éclairement et ce que l'on voit en fait sur une membrane circulaire de petites dimensions où les effets de bord sont très importants.

II. Amélioration de la capsule de mesure du récepteur du type de Golay. - II.1 PRÉPARATION DES SUPPORTS DE MEMBRANes. - II.1.1 Support de membrane réfléchissante. - La pièce métallique servant de support à la membrane miroir (Fig. $5 a$ ) est réalisée

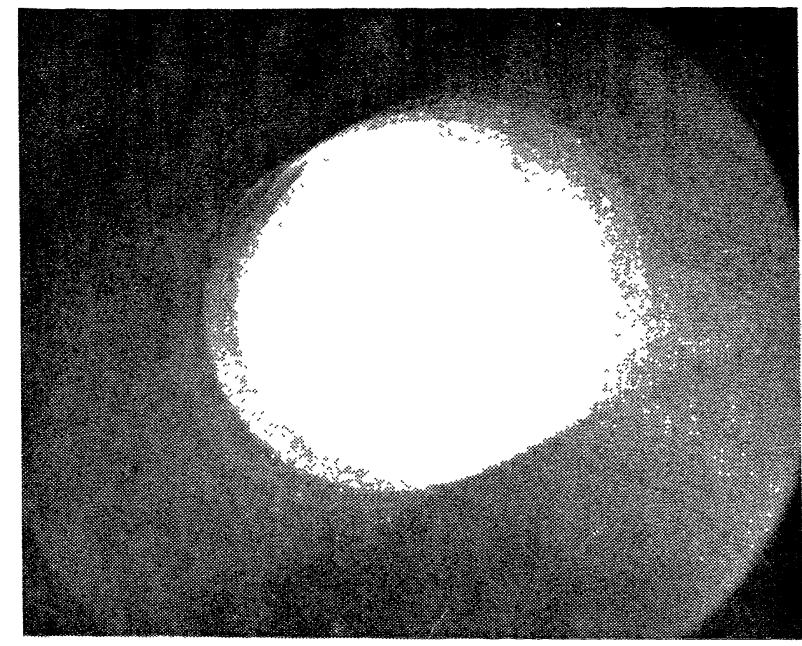

a)

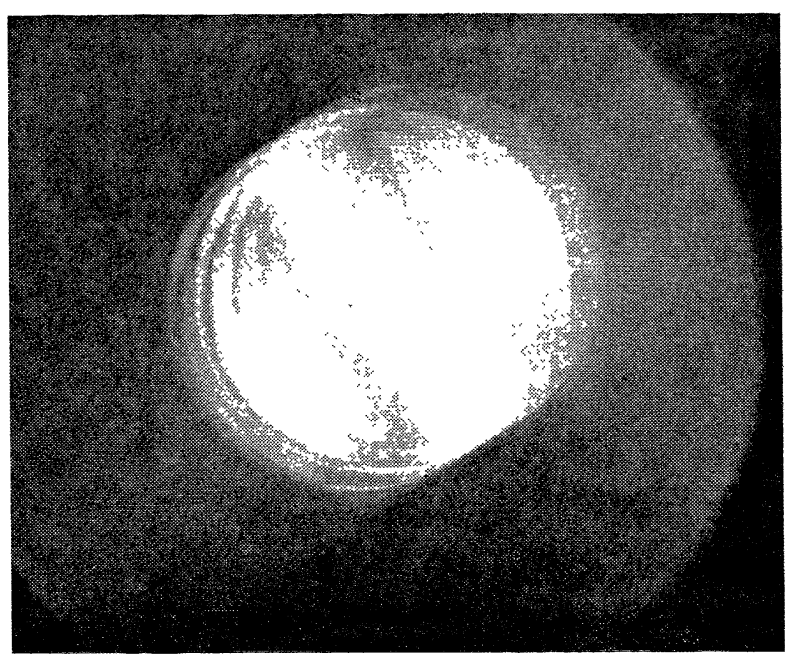

b)

FIG. 4. - Photographies des déformations de la membrane. faibles sur la figure $4 a$ et fortes sur la figure $4 b$.

en acier. Un soin tout particulier doit être apporté à la finition du bord inférleur de l'anneau no 1 . II doit être à angle vif. sans aucune bavure de métal. La partie plane de cet anneau doit être polie optiquement et ce travail demande beaucoup d'adresse car en le polissant on repousse des particules de métal qui rendent l'arête irrégulière.

Le meilleur surfaçage de l'anneau a été obtenu par frottement, à l'alcool tout d'abord. puis à sec sur une glace rectifiée. Lanneau $n^{\prime \prime} 2$ est nécessaire lors de la pose de la membrane. Il lui donne une tension uniforme sur toute sa partie utile. Après la pose on détruit la partie inutile de la membrane.

II 1.2 Support de membrane absorbante (Fig. 5b). - Le support est constitué par les limites de la cavité contenant une partie du gaz échauffé par la membrane. Après de nombreux essais plus ou moins empiriques faits par d'autres auteurs [1] [2]. la forme semi-sphérique a été adoptée. Ce support est réalisé en laiton poli et doré sur sa partie intérieure. 


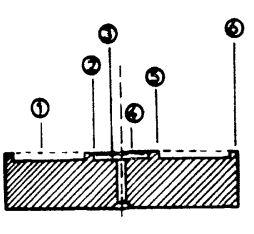

(1)

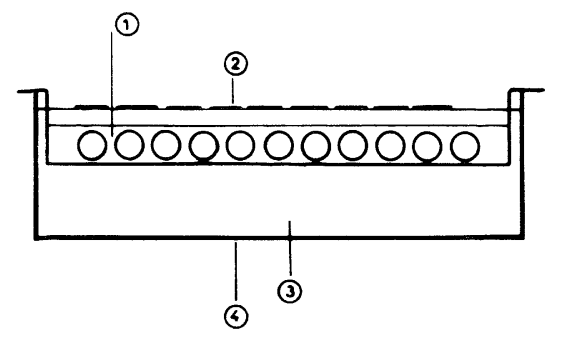

(c)

Fig. 5a. - Plan du support de la membrane réfléchissante : (1) Partie détruite après la pose de la membrane; (2) Bord intérieur; (3) Capsule manométrique; (4) Membrane réfléchissante ; (5) Anneau no 1 ; (6) Anneau no 2.

FIG. $5 b$. - Plan du support de la membrane absorbante : (1) Communication avec capsule manométrique et ballast; (2) Membrane absorbante ; (3) Xénon.

FIG. 5c. - Dispositif permettant d'obtenir les membranes de collodion : (1) Support de sortie des membranes; (2) Film de collodion; (3) Eau distillée ; (4) Cristallisoir.

II.2 Préparation des membranes (Fig. $5 c$ ). - II. 2.1 Membrane réfléchissante. - Les membranes sont réalisées à partir d'un film de collegedion obtenu en utilisant la méthode Grammont pour la fabrication d'écrans de télévision. La composition du collodion est la suivante :

6 g de nitrocellulose $12 \%$ Bergerac CA4E/130

75 g d'acétate d'isoamyle

$3,5 \mathrm{~cm}^{3}$ de phosphate tributylique

$2,5 \mathrm{~cm}^{3}$ d'alcool éthylique $96^{\circ}$

$1 \mathrm{~cm}^{3}$ de phtalate de butyle.

Après déshydratation de la nitrocellulose $\left(70^{\circ} \mathrm{C}\right.$ pendant 3 heures), les composants sont mélangés dans l'ordre énoncé ci-dessus. La solution obtenue est filtrée sous pression d'azote puis on la laisse reposer environ un mois. La membrane est obtenue en faisant tomber une goutte de collodion dans un cristallisoir rempli d'eau distillée. La surface de l'eau doit être exempte de poussière et avoir une température aussi voisine que possible de celle du collodion, de préférence aux environs de $10^{\circ} \mathrm{C}$. Le support permettant de recueillir les membranes, constituées de deux films soudés entre eux par capillarité, donne pour chaque goutte de collodion dix membranes utilisables. L'épaisseur de la membrane double, doit être voisine de $1000 \AA$; le film à la surface de l'eau doit donc avoir au maximum $500 \AA$ d'épaisseur. Cette épaisseur ne peut être estimée que quand la membrane est sèche, en observant ses couleurs d'interférence : son épaisseur doit être inférieure à $\lambda / 4$ pour la radiation violette (environ $0,4 \mu \mathrm{m}$ ), elle paraît blanche, légèrement teintée de jaune pâle. L'observation de la couleur de la membrane permet ainsi de faire une sélection rapide.

II.2.2 Membrane absorbante. - La fabrication des membranes absorbantes se fait à partir du même collodion et suivant le même procédé. Le collodion doit être un peu plus fluide de manière à obtenir une membrane dont l'épaisseur est comprise entre 100 et $500 \AA$, sans toutefois être trop mince, car après métallisation elle devient beaucoup trop fragile.

II.3 Contrôle Des membranes. - Nous avons utilisé une lunette métallographique Carl Zeiss Jéna permettant d'observer un objet opaque par réflexion sur fond clair ou sur fond noir. Le schéma de l'appareil (Fig. 6a) montre comment, avec le grandissement convenable, on peut, soit observer la membrane dans ses moindres détails, soit observer directement ses déformations et permettre ainsi un réglage direct et rapide du récepteur.

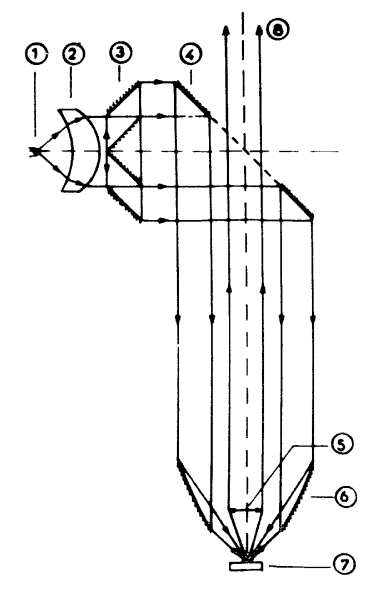

()

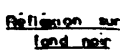

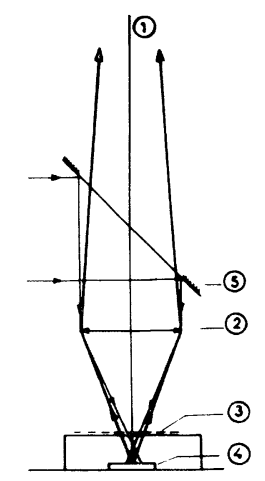

(b)
Fig. 6a. - Eclairage par réflexion sur fond noir : (1) Source ; (2) Condenseur ; (3) Miroirs coniques ; (4) Miroir plan annulaire ; (5) Objectif; (6) Miroir concave parabolique et annulaire; (7) Membrane ; (8) Oculaire.

FIG. 6b. - Contrôle de la membrane réfléchissante avec une grille : (1) Oculaire ; (2) Objectif ; (3) Grille ; (4) Membrane réfléchissante; (5) Miroir semi-réfléchissant.

Nous avons vu tout le soin qu'il est nécessaire d'apporter à la finition du support, aussi la membrane elle-même doit être d'une planéité parfaite, ses plus grands défauts étant inférieurs en grandeur aux déformations que l'on veut mesurer. On met en évidence les défauts de la membrane en l'observant à travers une grille placée à environ $1 \mathrm{~cm}$ au-dessus d'elle (Fig. 6b). On réalise ainsi un système optique analogue à un interféromètre de Mach-Zehnder dans lequel 
la présence de la grille fait apparaître dans le champ visuel une série de franges noires dont le parallélisme est affecté par des défauts de la membrane qui sont bien inférieurs à la longueur d'onde de la lumière permettant l'observation. La figure 7 est la photographie d'une membrane défectueuse sur son pourtour. Le contrôle des membranes absorbantes se fait après métallisation et le facteur mesuré est la transmission dans l'infrarouge lointain.

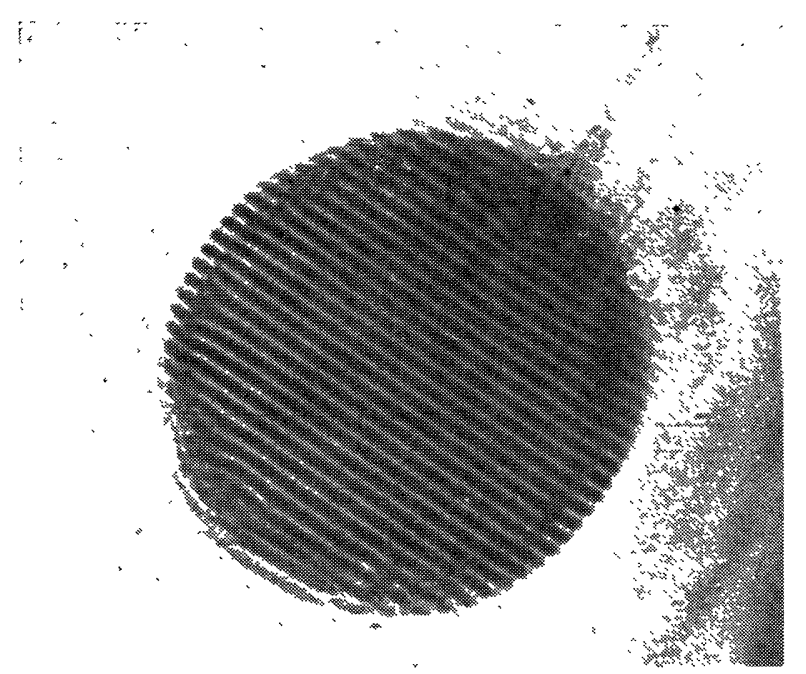

FIG. 7. - Photographie d'une membrane réfléchissante dont les défectuosités sont mises en évidence par le montage de la figure $6 b$.

II.4 MÉTAllisation DES MEMBRANES. - II.4.1 Membrane absorbante. - La membrane de collodion totalement transparente dans tout le domaine infrarouge est rendue partiellement absorbante par métallisation d'aluminium sous vide. Une lame absorbante très mince a un rendement maximum de $50 \%$ lorsqu'elle présente une transmission de $25 \%$ avec un pouvoir réflecteur de $25 \%$. De plus, lorsqu'on métallise un film de collodion, on constate que pour une pression de métallisation donnée, il existe une transmission constante pour toutes les longueurs d'onde. Nous avons vérifié ce phénomène [3] déjà observé dans le proche infrarouge, dans tout l'infrarouge. Les résultats de nombreuses mesures sont condensés dans la figure 8 sur laquelle on peut voir que la transmission non sélective d'une membrane varie linéairement avec le logarithme de la pression de métallisation. En réalité la transmission donnant les meilleurs résultats est plus grande, environ $30 \%$, car il faut tenir compte des réflexions successives sur les parois de la chambre. Le domaine de pression permettant d'obtenir une transmission correcte est hachuré sur la figure 8. Le collodion devant former les membranes absorbantes est déposé sur un support en acier comportant un trou rectangulaire d'environ $1 \mathrm{~cm}^{2}$. L'acier a été retenu car il permet de fixer magnétiquement les supports dans la cloche à métallisation. La quantité d'aluminium déposée est contrôlée par la mesure de

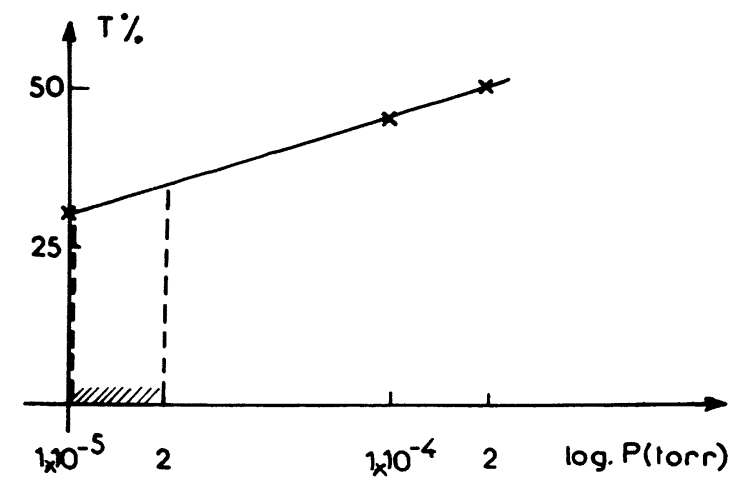

FIG. 8. - Variation de la transmission d'une membrane de collodion métallisée à l'aluminium en fonction du logarithme de la pression de métallisation.

la transmission d'une lame de collodion placée sur le faisceau lumineux éclairant une cellule au sulfure de cadmium LOR 04 (Radiotechnique) (Fig. 9a). Le logarithme de sa résistance est proportionnel au logarithme de son éclairement. Cette méthode de contrôle est une méthode comparative car les membranes utilisées et la membrane de mesure ne sont pas placées aux mêmes endroits et n'ont pas la même orientation: dans notre montage la membrane de mesure reçoit deux fois moins d'aluminium. Nous contrôlons la transmission des membranes ainsi réalisées dans le visible et l'infrarouge avec respectivement une cellule $\mathrm{CdS}$ et un banc d'optique qui n'envoie que les longueurs d'onde supérieures à $250 \mu \mathrm{m}$.

Après métallisation, avant même la rentrée d'air,

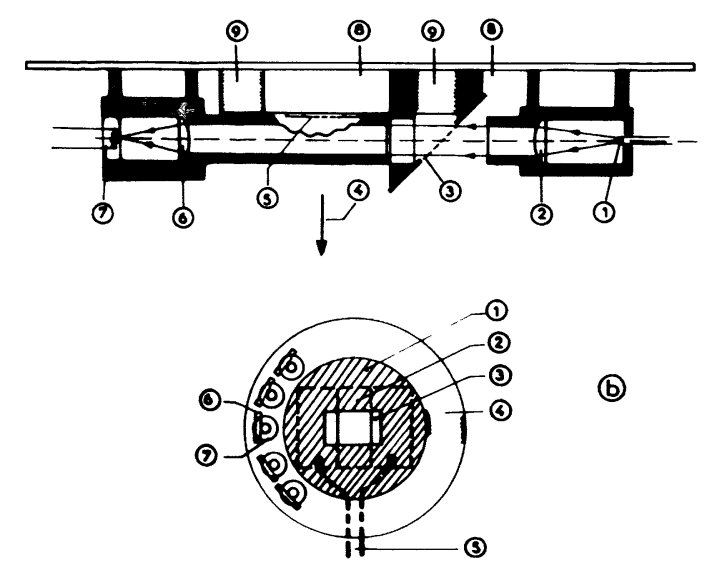

Fig. 9a. - Appareil de contrôle de la métallisation à l'aluminium : (1) Source lumineuse constante; (2) Lentille collimatrice; (3) Membrane de mesure placée à $45^{\circ}$; (4) Direction du creuset; (5) Membrane absorbante ; (6) Lentille objectif ; (7) Cellule CdS : LDR 04 ; (8) Pièces polaires supportant la membrane de mesure ; (9) Aimant en ferrite toroïdal.

Fig. 9b. - Dispositif de métallisation des membranes réfléchissantes : (1) Cache de la résistance témoin ; (2) Support en verre des électrodes; (3) Electrodes $\mathrm{A}$ et $\mathrm{B}$ en colle à l'argent ; (4) Aimant en ferrite dont les pôles se trouvent à l'intérieur et à l'extérieur du tore; (5) Ohmmètre; (6) Cale d'épaisseur ; (7) Support en acier de la membrane réfléchissante placée en biais sur la cale. 
l'aluminium déposé réagit avec les traces d'oxygène de la chambre à vide, réaction s'accélérant encore avec la rentrée d'air. La membrane initialement tendue se frippe et devient très fragile car l'aluminium réagit non seulement avec l'oxygène mais aussi avec le collodion (Fig. 10). Nous avons ainsi fabriqué une

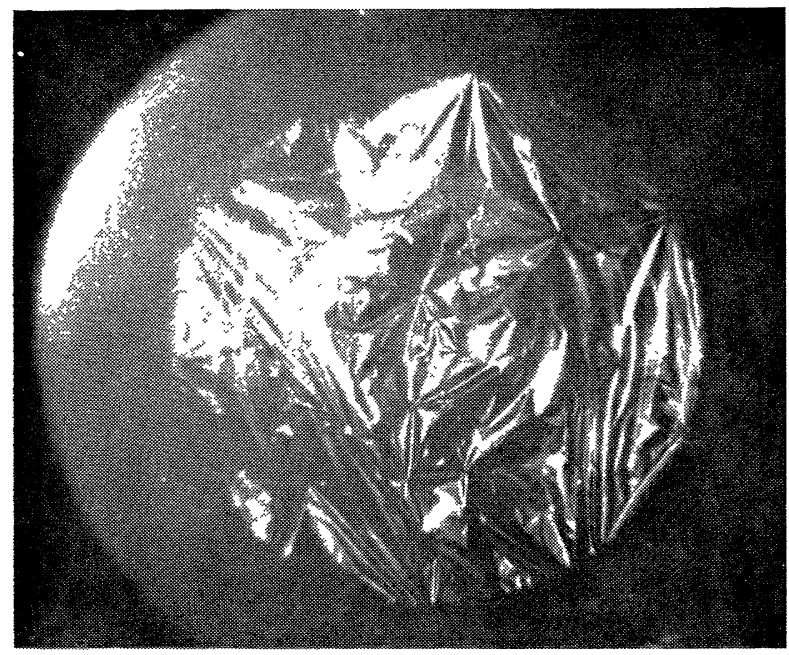

FIG. 10. - Photographie d'une membrane absorbante.

membrane absorbante de grande dimension qui peut être déposée sur six supports de membranes de récepteurs. Nous avons utilisé cette méthode, différente de celle des Etablissements UNICAM, car elle permet de déposer la métallisation à l'intérieur de la chambre du récepteur et d'améliorer le rendement de la membrane car son pouvoir réflacteur est plus faible (indice plus faible) du côté collodion que du côté métal (indice de valeur plus élevé). Les pertes du récepteur par rayonnement sont ainsi un peu diminuées et ses performances améliorées.

II.4.2 Membrane réfléchissante. - Les membranes réfléchissantes sont métallisées à l'antimoine, compromis entre un bon pouvoir réflecteur et une tension superficielle aussi faible que possible (Fig. 9b). La température de fusion de l'antimoine est de $630^{\circ} \mathrm{C}$, mais la pression de vapeur du métal est très importante avant sa fusion (Cf. Tableau I).

\begin{tabular}{|c|c|}
\hline \multicolumn{2}{|c|}{ TABLEAU I } \\
\hline$T\left({ }^{\circ} \mathrm{C}\right)$ & $P$ (torr) \\
\hline - & - \\
\hline 540 & $10^{-3}$ \\
\hline 620 & $10^{-2}$ \\
\hline 720 & $10^{-1}$ \\
\hline 840 & 1 \\
\hline 1440 & 760 \\
\hline
\end{tabular}

On voit donc que le métal se sublime dans le vide $\left(10^{-5}\right.$ torr) et qu'il n’est pas nécessaire de le fondre pour l'évaporer. ce qui permet de contrôler parfaitement la métallisation. La quantité de métal à déposer est celle qui est juste suffisante pour permettre de se rapprocher le plus possible de la valeur maximum $\mathrm{du}$ pouvoir réflecteur $(0,55$ pour le métal massif $)$ et celle qui affecte le moins la tension superficielle de la membrane non métallisée. Cette valeur se détermine par quelques essais préalables et se situe aux alentours de la valeur nécessaire pour donner une résistance carrée de 5 à $10 \mathrm{M} \Omega$ au moment de la métallisation. Il est important que la rentrée d'air se fasse très lentement car cette vitesse agit sur la cristallisation du dépôt métallique et par là même sur la souplesse de la membrane.

II.5 ReMPlissage EN XÉNON. - Le récepteur est rempli avec un gaz mono-atomique, de masse la plus élevée possible, le xénon (masse atomique 131,30). L'extrême sensibilité du récepteur aux variations de pression nous a conduit à imaginer le dispositif de la figure 11 permettant de faire le vide et de remplir en toute sécurité la capsule manométrique.

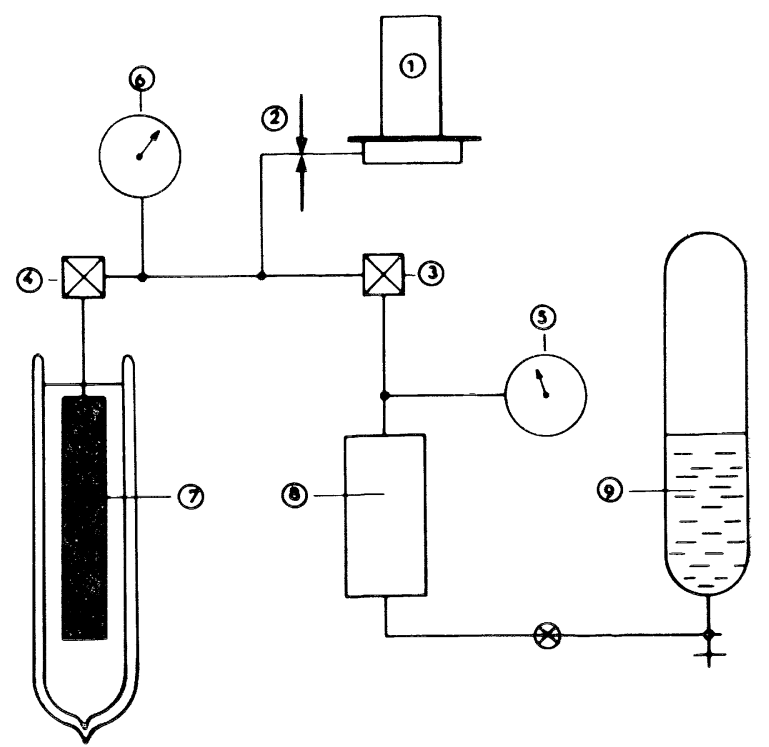

Fig. 11. - Schéma de l'installation de vide et de remplissage en xénon : (1) récepteur avec contrôle optique ; (2) Guillotine servant à obturer le capillaire; (3) Vanne pointeau : xénon; (4) Vanne pointeau : vide; (5) Pression de xénon; (6) Pression du récepteur; (7) Pompe à zéolithes; (8) Réservoir ballast de xénon; (9) Réservoir de xénon sous la pression critique de $60 \mathrm{~atm}$.

Pour connaître la pression optimale de xénon nous avons pu réaliser l'enregistrement continu de l'opération de vide et de remplissage en xénon dans un récepteur fonctionnant sur banc d'optique. Nous voyons (Fig. 12) que pour un récepteur contenant de l'air, le signal est une fonction linéaire et croissante de la pression alors que pour le xénon le signal présente un maximum pour une pression $P_{M}=600 \mathrm{~mm}$ de mercure. Nous avons tracé, pour un récepteur fictif (sans membrane), et pour des ouvertures croissantes de la vanne pointeau, les courbes de la pression 


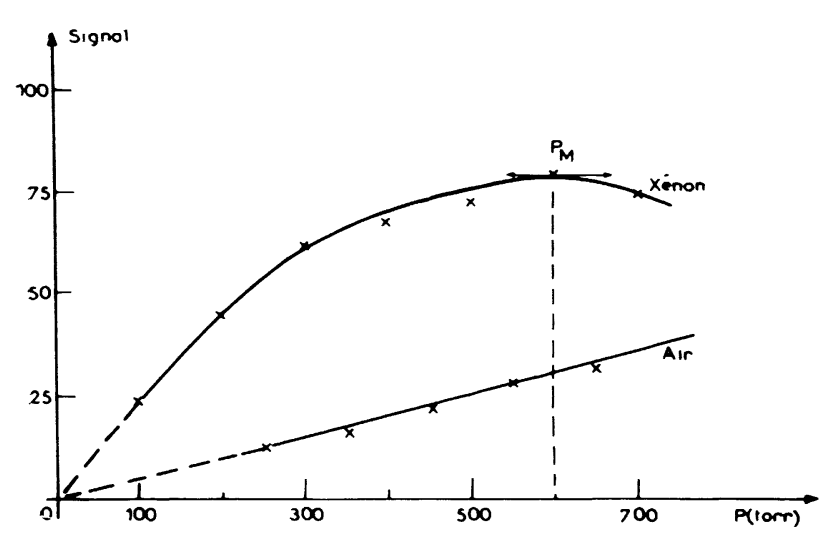

Fig. 12. - Signal détecté en fonction de la pression pour l'air et le xénon.

en fonction du temps (Fig. 13). Pour chaque courbe, la pente $\mathrm{d} P / \mathrm{d} t$ diminue au cours du temps et atteint une valeur $\mu$ assez faible pour que la membrane ne soit pas altérée. Le lieu des points où la pente est égale à $\mu$ délimite sur sa gauche le domaine interdit sous peine de détruire la membrane. Les opérations de pompage doivent se dérouler en suivant par exemple

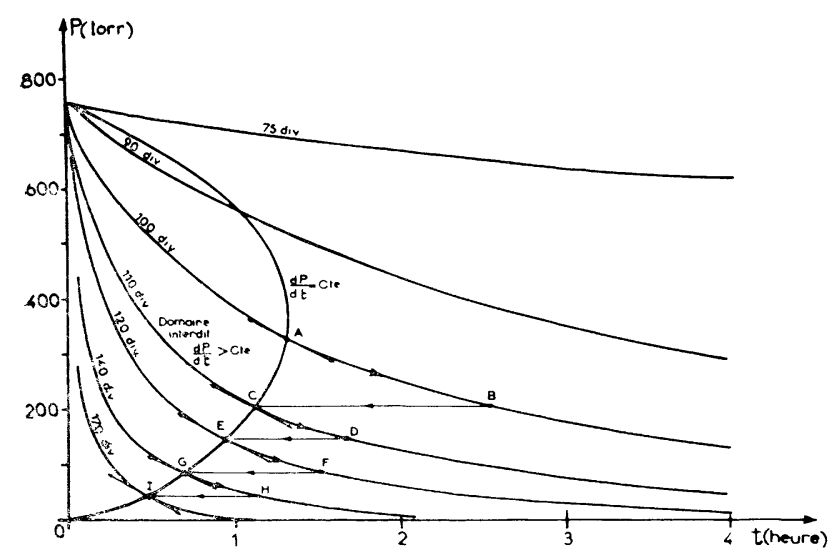

Fig. 13. - Variation de la pression dans un récepteur sans membrane en fonction du temps pour différentes ouvertures de la vanne pointeau.

le contour $\mathrm{ABCDEFGHI}$... et le temps de remplissage sera minimum si l'on peut programmer l'ouverture de la vanne pour suivre la courbe ACEGI tracée en trait épais sur la figure 13 où $\mathrm{d} P / \mathrm{d} t=$ constante $=\mu$. Dans le cas de la figure $(\mathrm{d} P / \mathrm{d} t)_{\max }=50$ torr $/ 19 \mathrm{~mm}$

$$
\text { et } t_{\min }=\frac{19 \mathrm{~mm} \times 760}{50}=288 \mathrm{~mm} \text {. }
$$

Pour chaque récepteur il existe une ouverture initiale de la vanne (repérée par environ 80 divisions sur la figure 13 donnant le débit) $(\mathrm{d} P / \mathrm{d} t)_{\max }=\mu$ que l'on contrôle par des déformations de la membrane. Il faut aussi tenir compte de la différence de viscosité de l'air et du xénon, dans l'écoulement des gaz à travers la vanne pointeau : le débit $G$ en $\mathrm{g} \mathrm{s}^{-1}$ suit la loi de Poiseuille car la pression ne devient jamais très faible (au mieux $10^{-2}$ torr) :

$$
G=\frac{\pi r^{4}}{8 \eta \mathrm{L}} \frac{\mathrm{PM}}{\mathrm{RT}} \cdot \Delta P .
$$

Pour l'air,

$(M / \eta)_{\mathrm{air}}=29 / 182,7 \# 0,16$, et pour le xénon $(M / \eta)_{\text {xenon }} \# 131 / 226=0,58$ soit $G_{\text {xe }} / G_{\text {air }}=3,6$.

Pour une même ouverture de la vanne, le remplissage en xénon sera 3,6 fois plus rapide qu'avec de l'air. Il faudra donc utiliser un autre réseau de courbes correspondant à des ouvertures plus faibles de la vanne pointeau.

III. Amélioration de la détection optique des déformations de la membrane. - Les variations d'éclairement de la membrane déformable sont enregistrées par une cellule photo-électrique photo-émissive VS 50 sensible dans le rouge (Fig. 14a). Le courant émis est
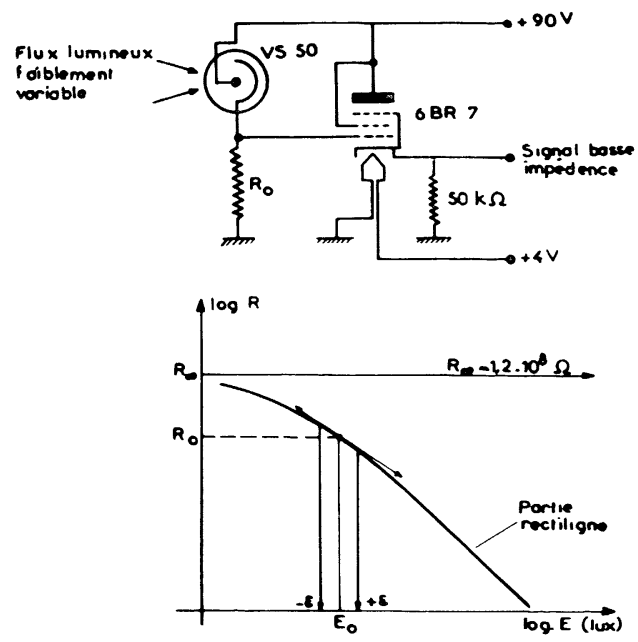

(b)

FIg. 14a. - Schéma de la détection électronique de la variation d'éclairement de la membrane déformante.

FIG. 14b. - Courbes d'une cellule ORP 90 dans des conditions de faible éclairement.

très faible et l'on utilise une résistance de charge $R_{0}=200 \mathrm{M} \Omega$. Cette valeur élevée produit un bruit Johnson non négligeable vis-à-vis de celui provenant de la cellule de mesure. Nous avons remplacé catte cellule à vide par une cellule photorésistante au sulfure de cadmium ORP 90 (Radiotechnique) (Fig. 14b). Pour respecter le fonctionnement du tube de sortie 6BR 7 et compte tenu de l'éclairement de la cellule nous avons été amenés à prendre $R_{0}=50 \mathrm{k} \Omega$. En effet la cellule présente une résistance de $2 \times 10^{5} \Omega$ correspondant à un éclairement moyen de $3 \times 10^{-2}$ lux. A cet éclairement faible correspond un point de fonctionnement situé dans une zone non linéaire du diagramme dynamique de la cellule mais nous ne mesurons que de très faibles variations $\pm \varepsilon$ de part et d'autre du point d'éclairement moyen $E_{0}$. Cette modification augmente de $15 \%$ le rapport Signal/Bruit. 
IV. Conclusions. - Nous avons réussi à porter récepteurs du commerce $\left(2,5 \times 10^{-11} \mathrm{~W} \mathrm{cps}^{-1 / 2}\right)$. la limite de sensibilité (NEP) aux environs de Par ailleurs la durée de vie est allongée, comprise $2 \times 10^{-11} \mathrm{~W} . \mathrm{cps}^{-1 / 2}$, valeur inférieure à celle des entre deux et trois ans.

\section{Bibliographie}

[1] Golay (M. J. E.), Theoretical consideration in heat and infrared detection, with particular reference to the pneumatic detector, Rev. Sci. Instr., 1947, $18,347$.

[2] Yoshinara (K.), Properties of pneumatic infrared detector. Sci. of Light, 1959, 8, 8.
[3] Brett (D. A.) and Sullivan (E. J.), Infrared transmittance of thin aluminum films on collodion substrates, J. Opt. Soc. Amer., 1956, 55, 1556.

[4] Hadni (A.), Essentials of Modern Physics applied to the Study of the Infrared, Pergamon Press Ltd, Oxford, 1967, 269. 